\title{
Some notes on vector fields in the tangent bundle
}

\author{
Kürsat Akbulut*1, Nejmi Cengiz ${ }^{1}$ and Furkan Yildirim ${ }^{2}$ \\ ${ }^{1}$ Department of Mathematics, Faculty of Science, Ataturk University, Erzurum, Turkey \\ ${ }^{2}$ Narman Vocational Training School, Ataturk University, Erzurum, Turkey
}

Received: 28 May 2019, Accepted: 25 October 2019

Published online: 26 December 2019.

\begin{abstract}
Let $(M, g)$ be a Riemannian manifold and $T(M)$ its tangent bundle with the horizontal lift ${ }^{H} \nabla$ of the affine connection $\nabla$ of $M$. The aims of the present paper are to study conditions of infinitesimal affine transformation for vertical and horizontal vector fields in the tangent bundle and to give necessary conditions for vertical, complete and horizontal vector fields in the tangent bundle to be a harmonic vector fields with respect to the horizontal lift ${ }^{H} \nabla$ of the affine connection $\nabla$ of $M$.
\end{abstract}

Keywords: Lift, Riemannian manifold, tangent bundle, infinitesimal Affine transformation, harmonic vector field.

\section{Introduction}

The theory of infinitesimal transformations was first given by Sophus Lie in [13]. In this work, he defined Lie groups and their accompanying Lie algebras and the identification of their role in geometry and especially the theory of differential equations. Afterwards, the term "Lie Algebra" as known the algebra of infinitesimal transformations of a Lie group was introduced in 1934 by Hermann Weyl. The problems of determining an infinitesimal affine transformation on $M$ and $T(M)$ have been considered by several authors. Yano and Ishihara, in [15], investigated an infinitesimal affine transformations on $T(M)$ with respect to the complete lift connection ${ }^{C} \nabla$. Hasegawa and Yamauchi, in [11], are improved the fiber-preserving hypothesis for non-affine infinitesimal projective transformations with horizontal lift connection.

Gezer and Akbulut, in [8] and [9], also studied infinitesimal affine transformations, geodesics and Killing vector fields on $T(M)$ with respect to the horizontal lift connections ${ }^{H} \nabla$. Akbulut and Salimov, in [3], investigated infinitesimal affine transformations on $T(M)$ with respect to synectic lift connection ${ }^{s} \nabla$. Similarly, Gezer and Akbulut, in [10], studied geodesics and Killing vector fields on $T(M)$ with respect to the Synectic lift connections ${ }^{s} \nabla$.

Investigation on harmonic vector fields is a topic which was studied when the tangent bundle is equipped with the $I I$, $I+I I$, Sasaki metric and g-natural metrics ([1], [2],[4],[5],[7],[12],[15]). Bejan and Duruta, in [4] and [5], studied the harmonic almost complex structure and harmonic connections with respect to the genaral natural metrics. Abbasi, Calvaruso and Perrone, in [1] and [2], investigated harmonicity of unit vector fields and harmonic sections of tangent bundle with respect to Riemannian $g$ - natural metrics. Similirly, Dragomir and Perrone, in [7], also studied harmonic vector fields.

The plan of the paper is as follows: In Section 2.1 we outline the necessary theory of tangent bundle to be applied later in the paper. In Sections 3.1 we study conditions of infinitesimal affine transformation for vertical and horizontal vector fields in the tangent bundle with respect to the horizontal lift ${ }^{H} \nabla$ of the Levi-Civita connection $\nabla$ of $M$.

\footnotetext{
*Corresponding author e-mail: kakbulut@ atauni.edu.tr
} 
In Sections 3.2 we give necessary conditions for vertical, complete and horizontal vector fields in the tangent bundle to be a harmonic vector fields with respect to the horizontal lift ${ }^{H} \nabla$ of the affine connection $\nabla$ of $M$.

\section{Preliminaries}

\subsection{Basic formulas on tangent bundles}

Let $M$ be a Riemannian manifold with metric $g$. We denote by $\mathfrak{I}_{q}^{p}(M)$ the set of all tensor fields of type $(p, q)$ on $M$. Manifolds, tensor field and connections are always assumed to be differentiable and of class $C^{\infty}$.

Let $T(M)$ be a tangent bundle of $M$ and $\pi$ the natural projection $\pi: T(M) \rightarrow M$. Let the manifold $M$ be covered by a system of coordinate neighbourhoods $\left(U, x^{i}\right)$, where $\left(x^{i}\right), i=1, \ldots, n$ is a local coordinate system defined in the neighbourhood $U$. Let $\left(y^{i}\right)$ be the Cartesian coordinates in each tangent space $T_{P}(M)$ at $p \in M$ with respect to the natural base $\left\{\frac{\partial}{\partial x^{i}}\right\}, p$ being an arbitrary point in $U$ whose coordinates are $\left(x^{i}\right)$. Then we can introduce local coordinates $\left(x^{i}, y^{i}\right)$ on the open set $\pi^{-1}(U) \subset T(M)$. We call them coordinates induced on $\pi^{-1}(U)$ from $\left(U, x^{i}\right)$. The projection $\pi$ is represented by $\left(x^{i}, y^{i}\right) \rightarrow\left(x^{i}\right)$. The indices $I, J, \ldots$ run from 1 to $2 n$, the indices $\bar{l}, \bar{j}$,run from $n+1$ to $2 n$.

Let $X=X^{i} \frac{\partial}{\partial x^{i}}$ be the local expression in $U$ of a vector field $X$ on $M$. Then the horizontal lift ${ }^{H} X$ and the vertical lift ${ }^{V} X$ of $X$ are given by,

$$
{ }^{V} X=X^{i} \partial_{\bar{l}}, \quad\left(\partial_{\bar{l}}=\frac{\partial}{\partial x^{\bar{l}}}\right)
$$

and

$$
{ }^{H} X=X^{i} \partial_{i}-y^{j} \Gamma_{j k}^{i} X^{k} \partial_{\bar{l}}
$$

with respect to the induced coordinates, where $\Gamma_{j k}^{i}$ are the coefficients of the Levi-Civita connection $\nabla$.

Suppose that we are given in $M$ a tensor field

$$
S=S_{l k \ldots j}^{i \ldots h} \frac{\partial}{\partial x_{i}} \otimes \ldots \otimes \frac{\partial}{\partial x_{h}} \otimes d x^{l} \otimes d x^{k} \otimes \ldots \otimes d x^{j},
$$

other than a function and a vector field $X=X^{i}\left(\frac{\partial}{\partial x^{i}}\right)$. We then define a tensor field $\gamma_{X} S$ in $\pi^{-1}(U)$ by

$$
\gamma_{X} S=\left(X^{l} S_{l k \ldots j}^{i \ldots h}\right) \frac{\partial}{\partial y^{i}} \otimes \ldots \otimes \frac{\partial}{\partial y^{h}} \otimes d x^{k} \otimes \ldots \otimes d x^{j}
$$

and a tensor field $\gamma S$ in $\pi^{-1}(U)$ by

$$
\gamma S=\left(y^{l} S_{l k \ldots j}^{i \ldots h}\right) \frac{\partial}{\partial y^{i}} \otimes \ldots \otimes \frac{\partial}{\partial y^{h}} \otimes d x^{k} \otimes \ldots \otimes d x^{j},
$$

with respect to the induced coordinates $\left(x^{h}, y^{h}\right), U$ being an arbitrary coordinate neighborhood in $M$ [15].

Let $M$ be a Riemannian manifold with metric $g$ whose components in a coordinate neighborhood $U$ are $g_{j i}$ and $\Gamma_{j i}^{h}$ the Christoffel symbols formed with $g_{j i}$. If in the neighborhood $\pi^{-1}(U)$ of the tangent bundle $T(M), U$ being neighborhood $M$, then ${ }^{H} g$ has components given by

$$
\left({ }^{H} g_{J I}\right)=\left(\begin{array}{cc}
\Gamma_{j}^{t} g_{t i}+\Gamma_{i}^{t} g_{j t} & g_{j i} \\
g_{j i} & 0
\end{array}\right)
$$


with respect to the induced coordinates $\left(x^{i}, y^{i}\right)$ in $T(M)$ and $\Gamma_{i}^{h}=y^{j} \Gamma_{j i}^{h}, \Gamma_{j i}^{h}$ being components of affine connection $\nabla$ in M. From (6), ${ }^{H} g$ has contravariant components

$$
\left({ }^{H} \tilde{g}^{I J}\right)=\left(\begin{array}{cc}
0 & g^{i j} \\
g^{i j} & -\Gamma_{h}^{j} g^{h i}-\Gamma_{h}^{i} g^{h j}
\end{array}\right)
$$

with respect to the induced coordinates in $T(M)$, where $g^{i j}$ denote the contravariant components of ${ }^{H} \tilde{g}$ in $M$, i.e., ${ }^{H} g_{I J}^{H} \tilde{g}^{I S}=$ $\delta_{J}^{S}[15]$.

Proposition 1. Let $g$ and $\nabla$ be, respectively, a pseudo-Riemannian metric and an affine connection such that $\nabla g=0$. Then ${ }^{H} \nabla^{H} g=0$, where the horizontal lift ${ }^{H} g$ of $g$ with respect to ${ }^{H} \nabla$ is a pseudo-Riemannian metric in $T(M)$ [15].

Proposition 2. Let $g$ be a pseudo-Riemannian metric and $\nabla$ the Riemannian connection determined by $g$ in $M$ such that $\nabla g=0$. Then, ${ }^{C} g$ coincides with the horizontal lift ${ }^{H} g$ of $g$ to $T(M)$ with respect to $\nabla[15]$.

Proposition 3. If we write $d s^{2}=g_{j i} d x^{j} d x^{i}$ the pseudo-Riemannian metric in $M$ given by $g$, then the pseudo-Riemannian metric in $T(M)$ given by the ${ }^{H} g$ of $g$ to $T(M)$ with respect to an affine connection $\nabla$ in $M$ is

$$
d s^{2}=2 g_{j i} \tilde{\delta} y^{j} d x^{i}
$$

where $\tilde{\delta} y^{j}=d y^{j}+\tilde{\Gamma}_{l k}^{j} y^{l} d x^{k}$ and $\tilde{\Gamma}_{j i}^{h}=\Gamma_{i j}^{h}$ are components of the connection $\tilde{\nabla}$ defined by

$$
\tilde{\nabla}_{X} Y=\nabla_{Y} X+[X, Y]
$$

for $X, Y \in \mathfrak{I}_{0}^{1}(M)[15]$

The horizontal lift ${ }^{H} \nabla$ of affine connection $\nabla$ in $M_{n}$ to $T(M)$ is defined by the conditions

$$
{ }^{H} \nabla_{V_{X}}{ }^{V} Y=0,{ }^{H} \nabla_{V_{X}}{ }^{H} Y=0,{ }^{H} \nabla_{H_{X}}{ }^{V} Y=\left(\nabla_{X} Y\right)^{V},{ }^{H} \nabla_{H_{X}}{ }^{H} Y=\left(\nabla_{X} Y\right)^{H},
$$

for $X, Y \in \mathfrak{I}_{0}^{1}(M)$. From (10) the horizontal lift ${ }^{H} \nabla$ of $\nabla$ has components ${ }^{H} \Gamma_{J I}^{K}$ such that

$$
\begin{gathered}
{ }^{H} \Gamma_{i j}^{k}=\Gamma_{i j}^{k},{ }^{H} \Gamma_{i \bar{j}}^{k}={ }^{H} \Gamma_{\bar{i} j}^{k}={ }^{H} \Gamma_{\bar{i} \bar{j}}^{k}={ }^{H} \Gamma_{\bar{i} \bar{j}}^{\bar{k}}=0, \\
{ }^{H} \Gamma_{i j}^{\bar{k}}=y^{s} \partial_{s} \Gamma_{i j}^{k}-y^{s} R_{s i j}^{k}{ }^{H} \Gamma_{\bar{l} j}^{\bar{k}}={ }^{H} \Gamma_{i j}^{\bar{k}}=\Gamma_{i j}^{k}
\end{gathered}
$$

with respect to the induced coordinates in $T\left(M_{n}\right)$, where $\Gamma_{i j}^{k}$ are components of $\nabla$ in $M$ [15].

Remark. The connection ${ }^{H} \nabla$ in Proposition 1 has nontrivial torsion even for the Riemannian connection $\nabla$ determined by $g$, unless $g$ is locally flat [15].

Explicit expressions for the Lie bracket [,] of the tangent bundle $T(M)$ are given in [6] and [15]. The Lie bracket of vertical and horizontal vector fields on $T(M)$ is given by the following propositions.

Proposition 4. Let $(M, g)$ be a pseudo-Riemannian manifold, $\nabla$ be a Levi-Civita connection. Then the Lie bracket on the tangent bundle $T(M)$ satisfies the following identities:

$$
\begin{aligned}
& {\left[{ }^{V} X,{ }^{V} Y\right]=0,} \\
& {\left[{ }^{H} X,{ }^{V} Y\right]={ }^{V}\left(\nabla_{X} Y\right),} \\
& {\left[{ }^{H} X,{ }^{H} Y\right]={ }^{H}[X, Y]-{ }^{V}(R(X, Y) y)}
\end{aligned}
$$


for all $X, Y \in \mathfrak{I}_{0}^{1}(M), R$ is the Riemannian curvature tensor of $\nabla$ defined by $R(X, Y)=\left[\nabla_{X}, \nabla_{Y}\right]-\nabla_{[X, Y]}$ and ${ }^{V}(R(X, Y) y)$ is a tensor field of type $(1,0)$ of $T(M)$ such that ${ }^{V}(R(X, Y) y)=\gamma(R(X, Y))$ which is locally expressed as $\gamma(R(X, Y))=$ $y^{s} R_{j k s}^{i} X^{j} Y^{k} \partial_{\bar{l}}$ with respect to the induced coordinates [6].

Let there be given an affine connections $\nabla$ and a vector field in $X \in \mathfrak{I}_{0}^{1}(M)$. Then the Lie derivative $L_{X} \nabla$ with respect to $X$ is, by definition, an element of $\mathfrak{I}_{2}^{1}(M)$ such that

$$
\left(L_{X} \nabla\right)(Y, Z)=L_{X}\left(\nabla_{Y} Z\right)-\nabla_{Y}\left(L_{X} Z\right)-\nabla_{[X, Y]} Z=\left[L_{X}, \nabla_{Y}\right] Z-\nabla_{[X, Y]} Z
$$

for any $Y, Z \in \mathfrak{I}_{0}^{1}(M)[14],[15]$.

In a manifold $M$ with affine connection $\nabla$, an infinitesimal affine transformation $x^{h^{\prime}}=x^{h}+X^{h}\left(x^{1}, \ldots, x^{n}\right) \delta t$ defined by a vector field $X \in \mathfrak{I}_{0}^{1}(M)$ is called an infinitesimal affine transformation if $L_{X} \nabla=0$, [15].

\section{Main results}

\subsection{Infinitesimal transformation in the tangent bundle}

Suppose that $X \in \mathfrak{I}_{0}^{1}(M)$, so that $X$ is a vector field in $M$. We define a vector field ${ }^{V} X$ in $T(M)$ by ${ }^{V} X(\iota \omega)=(\omega(X))^{V}$, where $\omega$ being an arbitrary 1 -form in $M$. Then, we call ${ }^{V} X$ the vertical lift of $X$ in $M$ to $T(M)$.

Additionally, let there be given an element $X$ of $\mathfrak{I}_{0}^{1}(M)$. Then, we define the horizontal lift ${ }^{H} X$ of $X$ by ${ }^{H} X=X^{C}-\nabla_{\gamma} X$ in $T(M)$ where $\nabla_{\gamma} X=\gamma(\nabla X)$.

Lemma 1. Let ${ }^{V} X$ and ${ }^{H} X$ be the vertical and horizontal lifts of $X$ to $T(M)$, respectively. Then

$$
\begin{gathered}
{ }^{H} \nabla_{\gamma(R(X, Y))}{ }^{V} Z=0, \\
{ }^{H} \nabla_{\gamma(R(X, Y))}{ }^{H} Z=0, \\
{ }^{H} \nabla_{V_{Y}} \gamma(R(X, Z))={ }^{V}(R(X, Z) Y), \\
{ }^{H} \nabla_{H_{Y}} \gamma(R(X, Z))=\gamma\left(\nabla_{Y} R(X, Z)\right)
\end{gathered}
$$

for any $X, Y, Z \in \mathfrak{I}_{0}^{1}(M)$, where $\gamma(R(X, Y))$ is a tensor field of type $(1,0)$ in $T(M)$.

Let $\nabla$ be a Riemannian connection in $M$ and the vertical lift $^{V} X$ of $X$ to $T(M)$. Using (10) and (12) in (13) we obtain

$$
\begin{aligned}
\left(L_{V_{X}}{ }^{H} \nabla\right)\left({ }^{V} Y,{ }^{H} Z\right) & =L_{V_{X}}\left({ }^{H} \nabla_{V_{Y}}{ }^{H} Z\right)-{ }^{H} \nabla_{V_{Y}}\left(L_{V_{X}}{ }^{H} Z\right)-{ }^{H} \nabla_{\left[{ }^{V} X,{ }^{V} Y\right]}{ }^{H} Z \\
& =-{ }^{H} \nabla_{V_{Y}}\left[{ }^{V} X,{ }^{H} Z\right] \\
& =-{ }^{H} \nabla_{V_{Y}}\left({ }^{V}[X, Z]-{ }^{V}\left(\nabla_{X} Z\right)\right) \\
& =-{ }^{H} \nabla_{V_{Y}}{ }^{V}[X, Z]+{ }^{H} \nabla_{V_{Y}}{ }^{V}\left(\nabla_{X} Z\right) \\
& =0 \\
\left(L_{V_{X}}{ }^{H} \nabla\right)\left({ }^{H} Y,{ }^{V} Z\right) & =L_{V_{X}}\left({ }^{H} \nabla_{H_{Y}}{ }^{V} Z\right)-{ }^{H} \nabla_{H_{Y}}\left(L_{V_{X}}{ }^{V} Z\right)-{ }^{H} \nabla_{\left[{ }^{V} X,{ }^{H} Y\right]}{ }^{V} Z \\
& =L_{V_{X}}{ }^{V}\left(\nabla_{Y} Z\right)-{ }^{H} \nabla_{H_{Y}}\left[{ }^{V} X,{ }^{V} Z\right]-{ }^{H} \nabla_{V[X, Y]-{ }^{V}\left(\nabla_{X} Y\right)} Z \\
& =\left[{ }^{V} X,{ }^{V}\left(\nabla_{Y} Z\right)\right]-{ }^{H} \nabla_{\left.V_{[X}, Y\right]} Z+{ }^{H} \nabla_{V}\left(\nabla_{X} Y\right){ }^{V}{ }^{V} \\
& =0,
\end{aligned}
$$




$$
\begin{aligned}
\left(L_{V_{X}}{ }^{H} \nabla\right)\left({ }^{V} Y,{ }^{V} Z\right) & =L_{V_{X}}\left({ }^{H} \nabla_{V_{Y}}{ }^{V} Z\right)-{ }^{H} \nabla_{V_{Y}}\left(L_{V_{X}}{ }^{V} Z\right)-{ }^{H} \nabla_{\left[{ }^{V} X,{ }^{V} Y\right]}{ }^{V} Z \\
& =-{ }^{H} \nabla_{V_{Y}}\left[{ }^{V} X,{ }^{V} Z\right] \\
& =0
\end{aligned}
$$

and

$$
\begin{aligned}
\left(L_{V_{X}}^{H} \nabla\right)\left({ }^{H} Y,{ }^{H} Z\right) & =L_{V_{X}}\left({ }^{H} \nabla_{H_{Y}}{ }^{H} Z\right)-{ }^{H} \nabla_{H_{Y}}\left(L_{V_{X}}{ }^{H} Z\right)-{ }^{H} \nabla_{\left[{ }^{V} X,{ }^{H} Y\right]}{ }^{H} Z \\
& =L_{V_{X}}{ }^{H}\left(\nabla_{Y} Z\right)-{ }^{H} \nabla_{H_{Y}}\left[{ }^{V} X,{ }^{H} Z\right]-{ }^{H} \nabla_{V_{[X, Y]}-{ }^{V}\left(\nabla_{X} Y\right)} Z \\
& =\left[{ }^{V} X,{ }^{H}\left(\nabla_{Y} Z\right)\right]-{ }^{H} \nabla_{H_{Y}}\left({ }^{V}[X, Z]-{ }^{V}\left(\nabla_{X} Z\right)\right)-{ }^{H} \nabla_{V[X, Y]}{ }^{H} Z+{ }^{H} \nabla_{V}\left(\nabla_{X} Y\right) \\
& { }^{H} Z \\
& ={ }^{V}\left[X, \nabla_{Y} Z\right]-{ }^{V}\left(\nabla_{X}\left(\nabla_{Y} Z\right)\right)-{ }^{H} \nabla_{H_{Y}}{ }^{V}[X, Z]+{ }^{H} \nabla_{H_{Y}}{ }^{V}\left(\nabla_{X} Z\right) \\
& ={ }^{V}\left[X, \nabla_{Y} Z\right]-{ }^{V}\left(\nabla_{X}\left(\nabla_{Y} Z\right)\right)-{ }^{V}\left(\nabla_{Y}[X, Z]\right)+{ }^{V}\left(\nabla_{Y}\left(\nabla_{X} Z\right)\right) \\
& ={ }^{V}\left(L_{X}\left(\nabla_{Y} Z\right)\right)-{ }^{V}\left(\nabla_{X}\left(\nabla_{Y} Z\right)\right)-{ }^{V}\left(\nabla_{Y}\left(L_{X} Z\right)\right)+{ }^{V}\left(\nabla_{Y}\left(\nabla_{X} Z\right)\right) \\
& \left.={ }^{V}\left(L_{X}\left(\nabla_{Y} Z\right)-\nabla_{X}\left(\nabla_{Y} Z\right)-\nabla_{Y}\left(L_{X} Z\right)\right)+\nabla_{Y}\left(\nabla_{X} Z\right)-\nabla_{[X, Y]} Z+\nabla_{[X, Y]} Z\right) \\
& ={ }^{V}\left(L_{X}\left(\nabla_{Y} Z\right)-\nabla_{Y}\left(L_{X} Z\right)-\nabla_{[X, Y]} Z-\left(\nabla_{X} \nabla_{Y} Z-\nabla_{Y} \nabla_{X} Z-\nabla_{[X, Y]} Z\right)\right) \\
& \left.\left.={ }^{V}\left(\left(L_{X} \nabla\right)(Y, Z)\right)-R(X, Y) Z\right)\right)
\end{aligned}
$$

for any $X, Y, Z \in \mathfrak{I}_{0}^{1}(M)$, where $R$ is curvature tensor of Riemannian connection of $\nabla$. Thus, from (14) we have,

Theorem 1. Let $\nabla$ be a Riemannian connection in $M$. If $X$ is an infinitesimal affine transformation of $M$ and $\nabla$ has zero curvature, then the vertical lift ${ }^{V} X$ of X to $T(M)$ is also infinitesimal affine transformation of $T(M)$ with respect to ${ }^{H} \nabla$.

Similarly, let $\nabla$ be a Riemannian connection in $M$ and the horizontal lift ${ }^{H} X$ of $X$ to $T(M)$. Using Lemma, (10) and (12) in (13) we have

$$
\begin{aligned}
& \left(L_{H_{X}}{ }^{H} \nabla\right)\left({ }^{H} Y,{ }^{V} Z\right)=L_{H_{X}}\left({ }^{H} \nabla_{H_{Y}}{ }^{V} Z\right)-{ }^{H} \nabla_{H_{Y}}\left(L_{H_{X}}{ }^{V} Z\right)-{ }^{H} \nabla_{\left[{ }^{H} X,{ }^{H}\right]}{ }^{V} Z \\
& =L_{H_{X}}{ }^{V}\left(\nabla_{Y} Z\right)-{ }^{H} \nabla_{H_{Y}}\left[{ }^{H} X,{ }^{V} Z\right]-{ }^{H} \nabla_{H}[X, Y]-\gamma(R(X, Y)){ }^{V} Z \\
& =\left[{ }^{H} X,{ }^{V}\left(\nabla_{Y} Z\right)\right]-{ }^{H} \nabla_{H_{Y}}{ }^{V}\left(\nabla_{X} Z\right)-{ }^{H} \nabla_{H[X, Y]}{ }^{V} Z+{ }^{H} \nabla_{\gamma(R(X, Y))}{ }^{V} Z \\
& ={ }^{V}\left(\nabla_{X} \nabla_{Y} Z\right)-{ }^{V}\left(\nabla_{Y} \nabla_{X} Z\right)-{ }^{V}\left(\nabla_{[X, Y]} Z\right) \\
& ={ }^{V}\left(\nabla_{X} \nabla_{Y} Z-\nabla_{Y} \nabla_{X} Z-\nabla_{[X, Y]} Z\right) \\
& ={ }^{V}(R(X, Y) Z), \\
& \left(L_{H_{X}}{ }^{H} \nabla\right)\left({ }^{V} Y,{ }^{V} Z\right)=L_{H_{X}}\left({ }^{H} \nabla_{V_{Y}}{ }^{V} Z\right)-{ }^{H} \nabla_{V_{Y}}\left(L_{H_{X}}{ }^{V} Z\right)-{ }^{H} \nabla_{\left[{ }^{H},{ }^{,} Y\right]}{ }^{V} Z \\
& =-{ }^{H} \nabla_{V_{Y}}\left[{ }^{H} X,{ }^{V} Z\right]-{ }^{H} \nabla_{\left[{ }^{H} X,{ }^{V} Y\right]}{ }^{V} Z \\
& \left.=-{ }^{H} \nabla_{V_{Y}}{ }^{V}\left(\nabla_{X} Z\right)-{ }^{H} \nabla_{V_{(}} \nabla_{X} Y\right){ }^{V} Z \\
& =0 \text {, } \\
& \begin{aligned}
\left(L_{H_{X}}{ }^{H} \nabla\right)\left({ }^{V} Y,{ }^{H} Z\right) & ={ }_{H_{X}}\left({ }^{H} \nabla_{V_{Y}}{ }^{H} Z\right)-{ }^{H} \nabla_{V_{Y}}\left(L_{H_{X}}{ }^{H} Z\right)-{ }^{H} \nabla_{\left[{ }^{H} X,{ }^{V} Y\right]}{ }^{H} Z \\
& \left.=-{ }^{H} \nabla_{V_{Y}}\left[{ }^{H} X,{ }^{H} Z\right]-{ }^{H} \nabla_{V_{(}} \nabla_{X} Y\right) \\
& =-{ }^{H} \nabla_{V_{Y}}\left({ }^{H}[X, Z]-\gamma(R(X, Z))-{ }^{H} \nabla_{H[X, Y]}{ }^{H} Z+{ }^{H} \nabla_{\gamma(R(X, Y))}{ }^{H} Z\right. \\
& ={ }^{V}(R(X, Z) Y),
\end{aligned}
\end{aligned}
$$


and

$$
\begin{aligned}
\left({ }^{L_{H}}{ }^{H} \nabla\right)\left({ }^{H} Y,{ }^{H} Z\right) & ={ }^{L_{H}}\left({ }^{H} \nabla_{H_{Y}}{ }^{H} Z\right)-{ }^{H} \nabla_{H_{Y}}\left(L_{H_{X}}{ }^{H} Z\right)-{ }^{H} \nabla_{\left[{ }^{H} X,{ }_{Y}{ }^{H}\right]}{ }^{H} Z \\
& ={ }^{H_{X}}{ }^{H}\left(\nabla_{Y} Z\right)-{ }^{H} \nabla_{H_{Y}}\left({ }^{H}\left(L_{X} Z\right)-\gamma(R(X, Z))\right)-{ }^{H} \nabla_{H}[X, Y]-\gamma(R(X, Y)){ }^{H} Z \\
& ={ }^{H}\left(L_{X}\left(\nabla_{Y} Z\right)\right)-\gamma\left(R\left(X, \nabla_{Y} Z\right)\right)-{ }^{H}\left(\nabla_{Y}\left(L_{X} Z\right)\right)+{ }^{H} \nabla_{H_{Y}} \gamma(R(X, Z))-{ }^{H} \nabla_{\left.H^{H} X, Y\right]}{ }^{H} Z+{ }^{H} \nabla_{\gamma(R(X, Y))}{ }^{H} Z \\
& ={ }^{H}\left(L_{X}\left(\nabla_{Y} Z\right)+\nabla_{Y}\left(L_{X} Z\right)-\nabla_{[X, Y]} Z\right)-\gamma\left(R\left(X, \nabla_{Y} Z\right)\right)+\gamma\left(\nabla_{Y} R(X, Z)\right) \\
& ={ }^{H}\left(\left(L_{X} \nabla\right)(Y, Z)\right)-\gamma\left(R\left(X, \nabla_{Y} Z\right)\right)+\gamma\left(\nabla_{Y} R(X, Z)\right)
\end{aligned}
$$

for any $X, Y, Z \in \mathfrak{I}_{0}^{1}(M)$, where $\gamma(R(X, Y))$ is a tensor field of type $(1,0)$ in $T(M)$ and $R$ is curvature tensor field of Riemannian connection of $\nabla$. Thus, from (15) we have

Theorem 2. Let $\nabla$ be a Riemannian connection in $M$. If $X$ is an infinitesimal affine transformation of $M$ and $\nabla$ has zero curvature, then the horizontal lift ${ }^{H} X$ of $X$ to $T(M)$ is also infinitesimal affine transformation of $T(M)$ with respect to ${ }^{H} \nabla$.

\subsection{Harmonic vector fields in the tangent bundle}

Let $\tilde{X}$ be a vector field in $T(M)$. The 1 -form $\omega$ defined by $\omega(\tilde{Y})=g(\tilde{X}, \tilde{Y}), \tilde{Y}$ being an arbitrary element of $\mathfrak{I}_{0}^{1}(T(M))$, is called the covector field associated with $\tilde{X}$ and denoted by $X^{*}$. If $\tilde{X}$ has local components $\tilde{X}^{A}$, then the associated convector field $\tilde{X}^{*}$ has local components

$$
\tilde{X}_{C}^{*}=\tilde{g}_{C B} \tilde{X}^{B}
$$

A vector field $X \in \mathfrak{I}_{0}^{1}(M)$ is said to be a Harmonic vector field of a Riemannian manifold with metric $g$, if $\nabla_{j} X_{i}-\nabla_{i} X_{j}=0$ and $\nabla_{i} X^{i}=0$, where $X_{j}=g_{j i} X^{i}$ are components of the covector field $X^{*}$ associated with $X$ and $\nabla$ is an affine connection of the metric $g$ [14].

We now consider a vector field $X \in \mathfrak{I}_{0}^{1}(M)$, then its vertical lift ${ }^{V} X \in \mathfrak{I}_{0}^{1}(T(M))$, complete lift ${ }^{C} X \in \mathfrak{I}_{0}^{1}(T(M))$ and horizontal lift ${ }^{H} X \in \mathfrak{I}_{0}^{1}(T(M))$ have respectively components of the form

$$
{ }^{V} X=\left(\begin{array}{l}
0 \\
X^{h}
\end{array}\right),{ }^{C} X=\left(\begin{array}{l}
X^{h} \\
y^{s} \partial_{s} X^{h}
\end{array}\right),{ }^{H} X=\left(\begin{array}{l}
X^{h} \\
-y^{s} \Gamma_{s i}^{h} X^{i}
\end{array}\right)
$$

with respect to the induced coordinates in $T(M)$, where $\Gamma_{i}^{h} x^{i}=y^{s} \Gamma_{s i}^{h} x^{i}[15]$.

Let $\tilde{X}$ be a vector field in $T(M)$ and $\left(\tilde{X}^{A}\right)=\left(\begin{array}{c}\tilde{X}^{h} \\ \tilde{X}^{\bar{h}}\end{array}\right)$ its components with respect to the induced coordinates. Then the covariant derivative ${ }^{H} \nabla \tilde{X}$ has components

$$
\begin{aligned}
& { }^{H} \nabla_{I} \tilde{X}^{J}=\partial_{I} \tilde{X}^{J}+{ }^{H} \Gamma_{I M}^{J} \tilde{X}^{M} \\
& { }^{H} \nabla_{I} \tilde{X}_{J}=\partial_{I} \tilde{X}_{J}-{ }^{H} \Gamma_{I J}^{M} \tilde{X}_{M}
\end{aligned}
$$

where ${ }^{H} \Gamma_{I M}^{J}$ being Christoffel symbols constructed with ${ }^{H} g$ and $\tilde{X}_{j}=\tilde{g}_{j i} \tilde{X}^{i}$ are components of the covector field $\tilde{X}^{*}$ associated with $\tilde{X}[15]$. 
Using (17) and (18) we see that, for the covariant derivatives of the vertical, complete and horizontal lifts of $\tilde{X}$ to $T(M)$ with the horizontal lift ${ }^{H} \nabla$ of the affine connection $\nabla$,

$$
\begin{aligned}
{ }^{H} \nabla_{I}{ }^{V} X^{J} & =\left(\begin{array}{cc}
0 & 0 \\
\nabla_{i} X^{j} & 0
\end{array}\right), \\
{ }^{H} \nabla_{I}{ }^{C} X^{J} & =\left(\begin{array}{cc}
\nabla_{i} X^{j} & 0 \\
y^{s} \partial_{s} \nabla_{i} X^{j}-y^{s} R_{s i h}^{j} X^{h} \nabla_{i} X^{j}
\end{array}\right), \\
{ }^{H} \nabla_{I}{ }^{H} X^{J} & =\left(\begin{array}{cc}
\nabla_{i} X^{j} & 0 \\
-\Gamma_{t}^{j} \nabla_{i} X^{t} & 0
\end{array}\right)
\end{aligned}
$$

with respect to the induced coordinates in $T(M)$. Thus, from (20) we have

Theorem 3. The vertical, complete and horizontal lifts of a vector field in $M$ to $T(M)$ with the horizontal lift ${ }^{H} \nabla$ of the affine connection $\nabla$ are parallel if and only if the vector field given in $M$ is parallel.

Using (16) and (17), we see that the covector fields respectively associated with ${ }^{V} X,{ }^{C} X$ and ${ }^{H} X$ have in $T(M)$ with the metric ${ }^{H} g$ components,

$$
{ }^{V} X_{I}=\left(X_{i}, 0\right),{ }^{C} X_{I}=\left(-\hat{X}_{i}+y^{s} \partial_{s} X_{i}, X_{i}\right),{ }^{H} X_{I}=\left(y^{s} \Gamma_{s i}^{t} X_{t}, X_{i}\right)
$$

with respect to the induced coordinates where $X_{j}=g_{j i} X^{i}$ are components of the covector field $\tilde{X}^{*}$ associated with $\tilde{X}$ and $\hat{X}_{j}=y^{s}\left(\nabla_{s} g_{k j}\right) g^{k t} X_{t}$.

Using (19) and (21) we obtain

$$
\begin{gathered}
{ }^{H} \nabla_{I}{ }^{V} X_{J}=\left(\begin{array}{cc}
\nabla_{i} X_{j} & 0 \\
0 & 0
\end{array}\right), \\
{ }^{H} \nabla_{I}{ }^{H} X_{J}=\left(\begin{array}{cc}
y^{s} \Gamma_{s j}^{t} \nabla_{i} X_{t} & \nabla_{i} X_{j} \\
0 & 0
\end{array}\right), \\
{ }^{H} \nabla_{I}{ }^{C} X_{J}=\left(\begin{array}{cc}
y^{s} \partial_{s} \nabla_{i} X_{j}+y^{s} R_{s i j}^{h} X_{h}-\nabla_{i} \hat{X}_{j} & \nabla_{i} X_{j} \\
\nabla_{i} X_{j}-\left(\nabla_{i} g_{h j}\right) g^{h t} X_{t} & 0
\end{array}\right) .
\end{gathered}
$$

From (22), we have

$$
\begin{aligned}
& { }^{H} \nabla_{I}{ }^{V} X_{J}-{ }^{H} \nabla_{J}{ }^{V} X_{I}=\left(\begin{array}{cc}
\nabla_{i} X_{j}-\nabla_{j} X_{i} & 0 \\
0 & 0
\end{array}\right),{ }^{H}{ }^{I J H} \nabla_{I}{ }^{V} X_{J}=0, \\
& { }^{H} \nabla_{I}{ }^{H} X_{J}-{ }^{H} \nabla_{J}{ }^{H} X_{I}=\left(\begin{array}{cc}
\Gamma_{j}^{t} \nabla_{i} X_{t}-\Gamma_{i}^{t} \nabla_{j} X_{t} & \nabla_{i} X_{j} \\
-\nabla_{j} X_{i} & 0
\end{array}\right),{ }^{H}{ }^{I J H} \nabla_{I}{ }^{H} X_{J}=g^{i j} \nabla_{i} X_{j},
\end{aligned}
$$

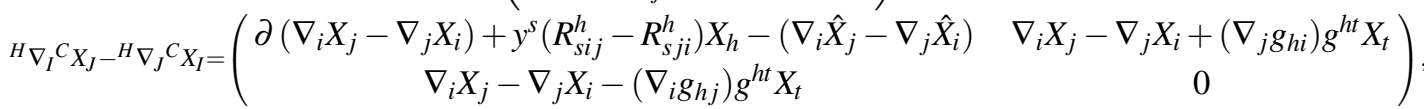

$$
\begin{aligned}
& { }^{H} g^{I J H} \nabla_{I}^{C} X_{J}=g^{i j} \nabla_{i} X_{j}+g^{i j}\left(\nabla_{i} X_{j}-\nabla_{i} g_{h j}\right) g^{h t} X_{t} \text {. }
\end{aligned}
$$

From (23), we have

Theorem 4. Necessary and sufficient conditions in order that (a) the vertical (b) complete and (c) horizontal lifts to T(M), with the metric ${ }^{H} g$, of a vector field $X$ in $M$ be a harmonic vector field in $T(M)$ are that, respectively, (a) Xis a harmonic vector field in $M ;(b) X$ is a parallel with vanishing second covariant derivative in $M$ and $\nabla$ is the affine connection of $M$ such that $\nabla g=0 ;($ c) $X$ is a parallel in $M$. 


\section{Acknowledgment}

The authors would like to express their gratitude to the anonymous referee for his (her) helpful comments and suggestions.

\section{Competing interests}

The authors declare that they have no competing interests.

\section{Authors' contributions}

All authors have contributed to all parts of the article. All authors read and approved the final manuscript.

\section{References}

[1] Abbassi, M.T.K., Calvaruso, G. and Perrone, D., Harmonicity of unit vector fields with respect to Riemannian g-natural metrics, Diff. Geom. Appl.27(2009) 157-169.

[2] Abbassi, M.T.K., Calvaruso, G. and Perrone, D., Harmonic sections of tangent bundles equipped with Riemannian g-natural metrics, Q. J. Math.62(2011), no. 2, 259-288.

[3] Akbulut, K. and Salimov, A.A. Infinitesimal affine transformations in a tangent bundle of a Riemannian manifold with affine connection ${ }^{s} \nabla={ }^{c} \nabla+{ }^{v} H$. Journal of Quality Measurement and Analysis, 2 (2006), no.1, 29-36.

[4] Bejan, C. L. and Druta, S. L., Connections which are harmonic with respect to general natural metrics, Diff. Geom. Appl.30(2012), no. 4, 306-317.

[5] Bejan, C. L. and Druta, S. L., Harmonic almost complex structures with respect to general natural metrics, Mediterranean Journal of Mathematics11(2014), no.1, 123-136.

[6] Dombrowski, P. On the geometry of the tangent bundle. J. Reine Angew. Math.,1962,1962(210),73-88.

[7] Dragomir, S., Perrone, D., Harmonic vector fields, Variational Principles and Differential Geometry, Elsevier, (2012).

[8] Gezer, A. and Akbulut, K. Infinitesimal affine transformations in the tangent bundle of a Riemannian manifold with respect to the horizontal lift of an affine connection. Hacettepe Journal of Mathematics and Statistics. 35 (2006), no. 2, 155-159.

[9] Gezer, A. and Akbulut, K. Infinitesimal automorphisms in the tangent bundle of a Riemannian manifold with horizontal lift of affine connection. Chiang Mai J. Sci., 34 (2007), no. 2, 151-159.

[10] Gezer, A. and Akbulut, K. Geodesics and Killing vector fields in a tangent bundle. Journal of Quality Measurement and Analysis, 2 (2006), no. 1, 115-121.

[11] Hasegawa, I. and Yamauchi, K. Infinitesimal holomorphically projective transformations on the tangent bundles with horizontal lift connection and adapted almost complex structure. J. Hokkaido Univ. Education, (2003), 53, 1-8.

[12] Ishihara, T., Harmonic sections of tangent bundles, J. Math. Tokushima Univ.,13 (1979), 23-27.

[13] Lie, S., The foundations of the theory of infinite continuous transformation groups - I, I. Treatise," Leipz. Ber. 1891, issue III, received 22-12-1891, pp. 316. Presented at the session on 8-6-1891. Gesammelte Abhandlungen, v. 6, art. XI, pp. 300-330.

[14] Yano K., Differential Geometry on Complex and Almost Complex spaces, The Mcmillan Company, New York (1965).

[15] Yano K. and Ishihara S. Tangent and cotangent bundles, Marcel Dekker, New York (1973). 